\title{
Spontaneous cervical hematoma associated with parathyroid adenoma
}

\author{
Habib U. Rehman MBBS, Milena Markovski MD, Amer Khalifa MD \\ Previously published at www.cmaj.ca
}

A 72-year-old woman presented with a sudden onset of pain, swelling and bruising of her neck that had occurred two days prior to presentation. A large bruise was observed over her neck (anterior and lateral) and anterior chest wall (Figure 1).

Results of blood investigations, including coagulation studies and hemoglobin, were normal, except for a corrected serum calcium level of $2.90(2.14-2.66) \mathrm{mmol} / \mathrm{L}$ and a parathyroid hormone level of 21.3 (1.3-6.8) ng/L. Computed tomography (CT) of the patient's neck and chest showed extensive ill-defined thickening involving the tissues of the left neck, but did not show the source of the hemorrhage (Appendix 1, available at www.cmaj.ca/cgi/content/full /cmaj.091167/DC1). Venous Doppler ultrasonography and CT angiogram of the neck were normal, as were an ultrasound of the thyroid and a sestamibi parathyroid scan. Given the elevated calcium and parathyroid hormone levels, the working diagnosis was extracapsular hemorrhage of a parathyroid adenoma.

At elective parathyroid exploration, a hard mass inferior to the left thyroid lobe was excised. Microscopic sections were consistent with parathyroid adenoma and focal hemosiderinladen macrophages were present, indicating hemorrhage (Appendix 1).

Extracapsular hemorrhage is a rare presentation of a parathyroid adenoma. Clues to the diagnosis include cervical pain when swallowing, cervical or thoracocervical ecchymosis and abnormal calcium levels. ${ }^{1}$ Other causes of this pattern of ecchymosis include trauma, dissecting aortic aneurysm and complications of medical procedures (e.g., cardiac catheterization, central line insertions).

Hemorrhage into a thyroid cyst is more common than hemorrhage into a parathyroid adenoma. Because the thyroid gland has a thicker capsule, hemotomas related to thyroid cysts are usually intracapsular and present as a palpable tender nodule in the neck. Intraparathyroid hemorrhage tears

From the Departments of Internal Medicine (Rehman), Family Medicine (Markovski) and Pathology (Khalifa), Regina Qu'Appelle Health Region, Regina, Sask.

CMAJ 2010. DOI:10.1503/cmaj.091167

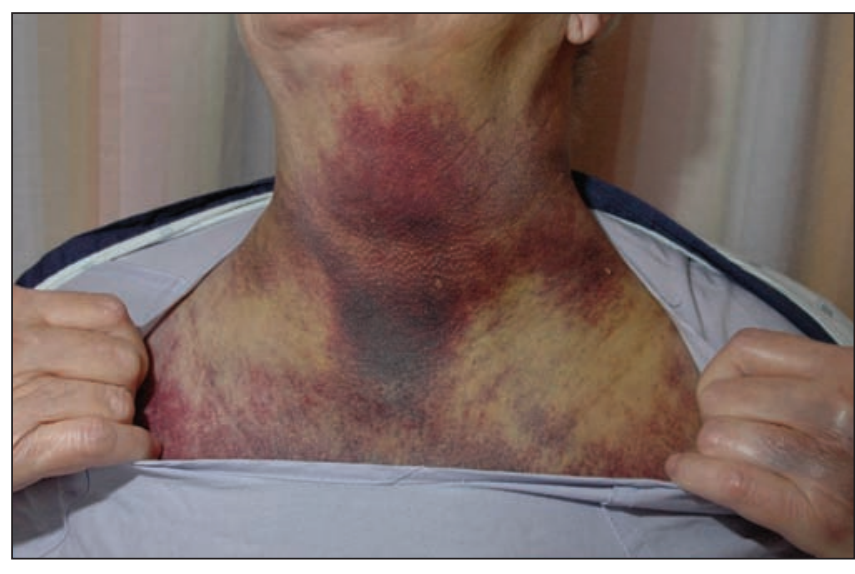

Figure 1: Extensive bruising of the anterior neck and chest in a 72-year-old woman.

through the capsule and produces a hematoma in the adjacent tissues, and may extend into the mediastinum. ${ }^{2}$

Potentially serious complications include rapidly progressive airway and esophageal obstruction, and acute blood loss. Acute hypercalcemia from release of parathyroid hormone from the damaged gland can occur, but if extensive parathyroid destruction has occurred, hypocalemia may be found. If the cause of a spontaneous cervical or mediastinal hematoma is unclear, it may be prudent to obtain a serum calcium level. In a stable patient, the initial treatment is conservative with referral for elective parathyroid exploration. ${ }^{3}$

This article has been peer reviewed.

Competing interests: None declared.

\section{REFERENCES}

1. Simcic KJ, McDermott MT, Crawford LM, et al. Massive extracapsular hemorrhage from a parathyroid cyst. Arch Surg 1989;124:1347-50.

2. Case records of the Massachusetts General Hospital. Weekly clinicopathological exercises. Case 22-1995. An 82-year-old woman with odynophagia and a large mediastinal mass. N Engl J Med 1995;333:175-81.

3. AACE/AAES Task Force on Primary Hyperparathyroidism. The American Association of Clinical Endocrinologists and the American Association of Endocrine Surgeons position statement on the diagnosis and management of primary hyperparathyroidism. Endocr Pract 2005;11:49-54. 